\title{
Spondylodiscitis Complicated by Chronic, Treatment-Dependent Nephrotic Syndrome
}

Aki Hamauchi, Tetsuhiko Yasuno* and Hitoshi Nakashima

Division of Nephrology and Rheumatology, Department of Internal Medicine, Fukuoka University, Fukuoka, Japan

*Corresponding author: Tetsuhiko Yasuno, Department of Pathology, Faculty of Medicine, Fukuoka University, Fukuoka, Japan, Tel: +81 92873 8008; E-mail: yasuno9584@fukuoka-u.ac.jp

Received date: January 22, 2015; Accepted date: March 11, 2015; Published date: March 16, 2015

Copyright: $\odot 2015$ Hamauchi A et al. This is an open-access article distributed under the terms of the Creative Commons Attribution License, which permits unrestricted use, distribution, and reproduction in any medium, provided the original author and source are credited.

\begin{abstract}
Spondylodiscitis develops in people with attenuated resistance to infection, such as patients undergoing steroid treatment and those with diabetes mellitus. Most patients with nephrotic syndrome are administered long-term steroids and immunosuppressants use, thus exposing patients to high risk of spondylodiscitis. However, no report has been published thus far on this issue. We here report a case of spondylodiscitis complicated by chronic, treatment-dependent nephrotic syndrome due to focal glomerular sclerosis.
\end{abstract}

Keywords: Spondylodiscitis; Treatment-dependent nephrotic syndrome

\section{Background}

Spondylodiscitis develops in people with attenuated resistance to infection, such as patients undergoing steroid treatment and those with diabetes mellitus [1]. Most patients with nephrotic syndrome are administered long-term steroids and immunosuppressants, thus exposing patients to high risk of spondylodiscitis [2,3]. However, no report has been published thus far on this issue. We here report a case of spondylodiscitis complicated by chronic, treatment-dependent nephrotic syndrome due to focal glomerular sclerosis.

\section{Case report}

The patient was a 53-year-old man who had been diagnosed in 2006 with focal glomerular sclerosis based on the histological findings of tissue obtained by a renal biopsy. He had been taking medications to treat pedal edema, proteinuria, and hematuria.

Oral prednisolone was administered, but the proteinuria was not attenuated. Therefore, the patient received steroid pulse and oral cyclosporine therapies. Thereby, the urinary protein levels decreased to $1 \mathrm{~g} /$ day. However, the nephrotic syndrome relapsed three times, and he was treated with low-density lipoprotein apheresis, which led him into remission in 2006.

Because of nephrotic syndrome relapsed, the patient was administered $40 \mathrm{mg}$ /day of steroids and $150 \mathrm{mg} /$ day of cyclosporine during two months. In May 2014, he experienced neck pain followed by an inability to move the lower extremities, and was transported to a hospital. Clinical examination revealed bilateral sensory paralysis at the Th2 and lower levels, as well as decreased muscle strength at the C6 and lower levels. An elevated inflammatory response was observed. Cervical spine magnetic resonance imaging revealed a low-signal intensity area in the epidural space between C3 and C5 (Figure 1). This finding led us to diagnose him with an epidural abscess. Cleaning and debridement of the anterior cervical spine and posterior cervical laminectomy was performed.Pus culture gathered during the operation revealed the presence of Staphylococcus aureus. Cefazolin sodium ( $6 \mathrm{~g} /$ day) was administered for two weeks until inflammation became resolved. Although administration of antibiotics resolved his inflammatory response, laminectomy did not resolve the paralysis.

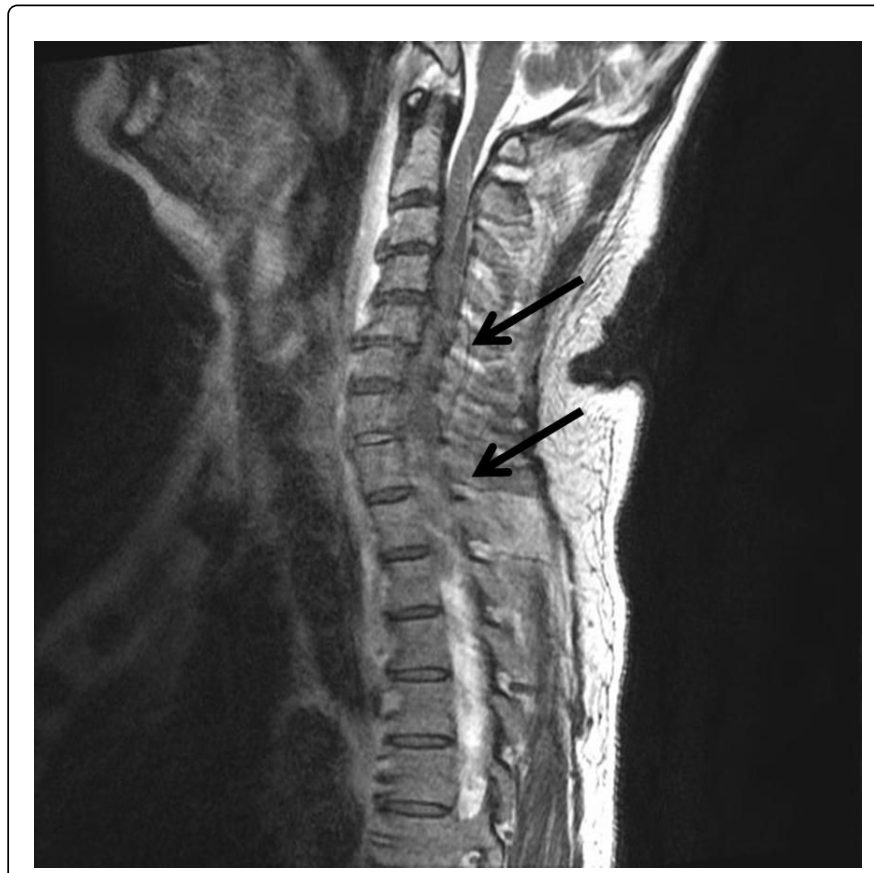

Figure 1: Magnetic resonance imaging revealing a low-signal intensity area in the epidural space between C3 and C7 (black arrows).

\section{Discussion}

Spondylodiscitis is a primary inflammation or infection of the intervertebral discs. It is thought that the direct hematogenous spread of infection into the disc is less common in adults due to the 
Citation: Hamauchi A, Yasuno T, Nakashima H (2015) Spondylodiscitis Complicated by Chronic, Treatment-Dependent Nephrotic Syndrome. J Neurol Neurophysiol 6: 276. doi:10.4172/2155-9562.1000276

Page 2 of 2

disappearance of vessels transversing the cartilage and plates, which usually occurs by age 7 [4]. Several factors may increase a patient's risk for developing cervical spondylodiscitis, including diabetes mellitus, steroid therapy, chronic disease, immunosuppression, and advanced age $[5,6]$. The most common causative bacterial pathogen in spondylodiscitis is Staphylococcus aureus [5]. In this case, the patient had a history of recurrent nephrotic syndrome, steroid treatment, and decayed teeth. The steroid doses were increased after each relapse. As a result, high doses of steroids and cyclosporine were administered over a long period. These conditions may have been contributing factors to the development of spondylodiscitis.

Generally, common spondylodiscitis alone does not cause tetraplegia. Anterior debridement of the cervical spine and posterior cervical laminectomy should be performed within 24 hours, which is considered useful for avoiding permanent disorder. However, in this case, the epidural abscess may have spread to the spinal canal, rapidly causing tetraplegia below the neck; unfortunately, the operation did not resolve the paralysis.

We measured blood concentration of cyclosporine and the area under the blood concentration curve (AUC) kept 2,000 ng/h/mL, Cmax $700 \mathrm{ng} / \mathrm{mL}$. The patient had been taking steroids and cyclosporine for 8 years, with the dosage being increased or decreased depending on whether nephrotic syndrome relapsed. The Kidney Disease Initiative Global Outcome (KDIGO) guidelines recommend that the dosage of immunosuppressive drugs, such as steroids, should be equal to that of cyclosporine, cyclophosphamide, or tacrolimus prescribed in primary care. Although administration of steroids and cyclosporine is recommended for chronic, treatment-dependent nephrotic syndrome, a clear limit for the period of administration has not been established. When frequent relapses occur and renal function remains unchanged, as in the case described in this report, immunosuppressants are typically administered in high doses for a long period, which increases the likelihood of spondylodiscitis and epidural abscess. Spinal cord compression is a surgical emergency. When a patient undergoes treatment for nephrotic syndrome and is in an immunosuppressed condition, caution should be exercised because of the possibility of developing severe spondylodiscitis, which, albeit rare, is difficult to diagnose.

\section{References}

1. Krogsgaard MR, Wagn P, Bengtsson J (1998) Epidemiology of acute vertebral osteomyelitis in Denmark: 137 cases in Denmark 1978-1982, compared to cases reported to the National Patient Register 1991-1993. Acta Orthop Scand 69: 513-517.

2. Rezai AR, Woo HH, Errico TJ, Cooper PR (1999) Contemporary management of spinal osteomyelitis. Neurosurgery 44: 1025-1026.

3. Weinstein MA, Eismont FJ (2005) Infections of the spine in patients with human immunodeficiency virus. J Bone Joint Surg Am 87: 604-609.

4. Rudert M, Tillmann B (1993) Lymph and blood supply of the human intervertebral disc. Cadaver study of correlations to discitis. Acta Orthop Scand 64: 37-40.

5. Anton K, Christoph R, Cornelius FM (1999) Osteomyelitis and pathological fracture of the axis. Case illustration. J Neurosurg 90: 162.

6. Spuck S, Arnold H, Kranz R, Solbach W, Kaemmerer R(2005) Rapid manifestation of cervical vertebral osteomyelitis. Acta Neurochir 147: 671-673. 